\title{
Reduced polyphenol oxidase gene expression and enzymatic browning in potato (Solanum tuberosum L.) with artificial microRNAs
}

Ming Chi ${ }^{1,2}$, Basdeo Bhagwat ${ }^{2}$, W David Lane ${ }^{2}$, Guiliang Tang ${ }^{3}$, Yinquan Su', Runcang Sun ${ }^{1}$, B Dave Oomah ${ }^{2}$, Paul A Wiersma ${ }^{2}$ and Yu Xiang ${ }^{2^{*}}$

\begin{abstract}
Background: Polyphenol oxidase (PPO), often encoded by a multi-gene family, causes oxidative browning, a significant problem in many food products. Low-browning potatoes were produced previously through suppression of PPO gene expression, but the contribution of individual PPO gene isoform to the oxidative browning process was unknown. Here we investigated the contributions of different PPO genes to total PPO protein activity, and the correlations between PPO protein level, PPO activity and tuber tissue browning potential by suppression of all previously characterized potato PPO genes, both individually and in combination using artificial microRNAs (amiRNAs) technology.

Results: Survey of the potato genome database revealed 9 PPO-like gene models, named StuPPO1 to StuPPO9 in this report. StuPPO1, StuPPO2, StUPPO3 and StuPPO4 are allelic to the characterized POTP1/P2, POT32, POT33 and POT72, respectively. Fewer ESTs were found to support the transcriptions of StuPPO5 to StuPPO8. StuPPO9 related ESTs were expressed at significant higher levels in pathogen-infected potato tissues. A series of browning phenotypes were obtained by suppressing StuPPO1 to StuPPO4 genes alone and in combination. Down-regulation of one or several of the PPO genes did not usually cause up-regulation of the other PPO genes in the transgenic potato tubers, but resulted in reduced PPO protein levels. The different PPO genes did not contribute equally to the total PPO protein content in the tuber tissues, with StuPPO2 accounting for $\sim 55 \%$ as the major contributor, followed by StuPPO1, 25-30\% and StuPPO3 and StuPPO4 together with less than 15\%. Strongly positive correlations between PPO protein level, PPO activity and browning potential were demonstrated in our analysis. Low PPO activity and low-browning potatoes were produced by simultaneous down-regulation of StuPPO2 to StuPPO4, but the greatest reduction occurred when StuPPO1 to StuPPO4 were all suppressed.
\end{abstract}

Conclusion: StUPPO1 to StuPPO4 genes contributed to browning reactions in tuber tissues but their effect was not equal. Different PPO genes may be regulated independently reflecting their diversified functions. Our results show that amiRNAs can be used to suppress closely related members of highly conserved multi-gene family. This approach also suggests a new strategy for breeding low-browning crops using small DNA inserts.

Keywords: Artificial microRNA (amiRNA), Enzymatic browning, Polyphenol oxidase gene family, Solanum tuberosum L

\footnotetext{
* Correspondence: yu.xiang@agr.gc.ca

${ }^{2}$ Agriculture and Agri-Food Canada, Pacific Agri-Food Research Centre,

Summerland, British Columbia VOH 1Z0, Canada

Full list of author information is available at the end of the article
} 


\section{Background}

Polyphenol oxidase (PPO) is nearly ubiquitous in angiosperms and belongs to a class of copper-binding enzymes that catalyze the oxidation of phenolics to quinones. The subsequent non-enzymatic polymerization of the quinones leads to formation of brown pigments that are the cause of post-harvest deterioration and loss of quality in many economically important crops [1,2]. Losses caused by the browning resulting from $\mathrm{PPO}$ catalyzed-oxidations probably account for $50 \%$ of the losses of industrial production of fruits and vegetables [3]. Several reports have described reduced browning reaction in crops by suppression of PPO gene expression using transgenic transformation with PPO gene fragments in configurations such as sense, antisense or double-stranded RNA [4-10]. Those approaches functioned by establishing an RNA silencing mechanism guided by a population of heterogeneous small interfering RNAs (siRNAs) [11]. Inevitably, the whole PPO gene family in the transgenic hosts was targeted because PPO genes are members of a multi-gene family with highly conserved gene sequences [2]. Because of this, it has been difficult to assess the contribution made by the individual PPO gene(s) to the oxidative browning process in different tissues. In addition to the undesired browning activity, PPOs appear to play important roles in signal transduction, stress and defense response throughout plant growth and development, but the specific PPO gene members involved in the different functions has not been elucidated. In potato (Solanum tuberosum L.), five PPO genes, namely POTP1 (GenBank ID: M95196), POTP2 (M95197), POT32 (U22921), POT33 (U22922) and POT72 (U22923), were previously identified and characterized $[1,12]$. The nucleotide sequences of POTP1 and POTP2 are over 97\% identical. POTP1/P2, POT32, POT33 and POT72 share 70-82\% nucleotide homology. A previous study based on RNA Northern blot analysis revealed that POTP1 and POTP2 genes were expressed mainly in potato leaves and flowers, POT32 and POT33 mRNA were detected mainly in tubers with the POT32 being the major form throughout tuber development, and POT72 gene was mainly expressed in the roots [1].

Artificial microRNA (amiRNA) technology is a newly developed approach for inducing loss of gene function in plants [13-16]. It utilizes microRNA (miRNA) gene backbones to express artificial small RNAs that are usually 21 nucleotides (nt) in length. The resultant amiRNAs join in RNA silencing pathways and guide silencing of the gene of interest [17]. One of the advantages of amiRNA strategy is that it generates a single type of small RNA population all with the same selective nucleic acid sequence. It provides a feasible method for either silencing an individual gene or simultaneously silencing or partially silencing a multi-gene family while at the same time minimizing the risk of unpredicted off-target effects [18]. The amiRNA strategy has been applied in functional genetics studies using model plants, such as Arabidopsis and also agricultural crops, such as rice, alfalfa and tomato in recent years [19-21]. However, there were fewer reports of the targeting of closely related members of multi-gene families $[20,22]$.

Here we reported suppression of the characterized members of the PPO gene family, i.e. POTP1/P2, POT32, POT33 and POT72, individually or in combination in potato using amiRNAs. This allowed us to investigate the contributions of the different PPO genes to the total PPO protein activity in potato tubers and to further understand the correlations between PPO protein level, PPO activity and tuber tissue browning potential. Our results show that amiRNAs can be applied to suppress the expression of individual members of a highly conserved gene family. A series of browning phenotypes resulted from the suppression of different PPO gene isoforms in potato. Our results also suggest a new strategy for developing low-browning or non-browning crops.

The PPO gene suppression research in this report started before the availability of the potato genome sequence data, but we recently surveyed the PPO gene family in the potato genome and discovered 9 PPO-like gene models. The PPO gene models are systematically named as StuPPO1 to StuPPO9 (Solanum tuberosum polyphenol oxidase 1 to 9). POTP1/P2, РОT32, РОТ33 and POT72 are considered allelic to StuPPO1, StuPPO2, StuPPO3 and StuPPO4, respectively. For continuity in the systematic nomenclature in this report, we renamed POTP1/P2, POT32, POT33 and POT72 to StuPPO1, StuPPO2, StuPPO3 and StuPPO4.

\section{Results}

\section{Genome-wide survey of PPO-like gene models in Solanum tuberosum}

A genome-wide search of the recent $S$. tuberosum whole genome database in the US Joint Genome Institute (http://www.jgi.doe.gov) reveals 9 PPO-like gene models, tentatively named StuPPO1 to StuPPO9 in this report (Table 1, Additional file 1). The StuPPO1 to StuPPO8 genes are aligned on chromosome 8, and StuPPO9 is located on chromosome 2. StuPPO1 and StuPPO6 are in close proximity to each other in a 47-kb region, while StuPPO2, StuPPO3, StuPPO4, StuPPO5, StuPPO7 and StuPPO8 are clustered in tandem in a 144-kb region. The two regions are separated by a distance of 2,072-kb on chromosome 8 . Analysis of the deduced amino acid sequences of the major peptides encoded by the PPO-like genes suggests that the predicted proteins all contain three typical PPO protein domains: the tyrosinase domain (pfam00264), the PPO1_DWL domain (pfam12142) and 
Table 1 List of predicted potato PPO gene models

\begin{tabular}{lllll}
\hline $\begin{array}{l}\text { Tentative gene } \\
\text { name (in this report) }\end{array}$ & Locus location & $\begin{array}{l}\text { Number of } \\
\text { intron }\end{array}$ & $\begin{array}{l}\text { Predicted transcript name } \\
\text { assigned by potato genome } \\
\text { sequencing consortium (PSGC) }\end{array}$ & $\begin{array}{l}\text { Possible Allele (GenBank ID, } \\
\text { nucleotide sequence identity\%) }\end{array}$ \\
\hline StuPPO1 & chr08, 30458794..30460741 & 0 & PSGC003DMT400076054 & $\begin{array}{l}\text { POTP1 (M95196, 97.3\%)/POTP2 (M95197, } \\
\text { 97.9\%)/XM_006355177 (100\%) } \\
\text { StuPPO2 }\end{array}$ \\
StuPPO3 & chr08, 32672194..32674192 & 0 & PSGC0003DMT400048684 & POT32 (U22921, 96.7\%)/XM_006365321 (95.5\%) \\
& chr08, 32687330..32689280 & 0 & PSGC0003DMT400048681 & POT33 (U22922, 94.7\%)/XM_006365320 \\
StuPPO4 & chr08, 32667904..32669792 & 0 & PSGC0003DMT400048685 & POT72 (U22923, 96.8\%) \\
StuPPO5 & chr08, 32591830..32593339 & 0 & PSGC0003DMT400048692 & XR_183056 (84.6\%) \\
StuPPO6 & chr08, 30504049..30505788 & 0 & PSGC0003DMT400076055 & XM_004245989 (89.1\%) \\
StuPPO7 & chr08, 32577708..32579291 & 0 & PSGC0003DMT400048703 & XR_183056 (84.8\%) \\
StuPPO8 & chr08, 32703818..32721729 & 2 & PGSC0003DMT400048679 & XM_006365329 (100\%) \\
StuPPO9 & chr02, 55593718..55596019 & 1 & not available & XM_006347021 (100\%) \\
\hline
\end{tabular}

the PPO1_KFDV domain (pfam12143) [23,24], but the tyrosinase domains with the putative StuPPO5 and StuPPO7 peptides are shorter than the others and incomplete (Additional file 1: Part B). StuPPO1, StuPPO2, StuPPO3 and StuPPO4 are possibly allelic to the characterized POTP1/P2, POT32, POT33 and POT72, respectively, considering that the nucleotide sequences between the potential alleles are $95-99 \%$ identical (Table 1, Additional file 1). Numerous ESTs were found from different developmental potato tissues for StuPPO1 to StuPPO4 (Additional file 1: Part A and Part C). Surprisingly, StuPPO1 appears to be the only possible allele to the POTP1 and POTP2 genes, and no duplication of the StuPPO1 locus was observed by searching the $S$. tuberosum genome sequence. StuPPO5, StuPPO6 and StuPPO7 are three novel PPO-like gene models predicted from this $S$. tuberosum genome sequence analysis. However, only few EST fragments ( 0 to 3 ) that probably relate to the potential transcripts of these three genes were found, and the ESTs cover only fragmental regions of the putative transcripts (Additional file 1: Part A and Part C). The low prevalence in EST databases suggests that StuPPO5, StuPPO6 and StuPPO7 may be expressed at very low levels in S. tuberosum. StuPPO8 and StuPPO9 are the only PPOlike gene models with introns. No EST from $S$. tuberosum EST databases was found for StuPPO8, suggesting that this gene sequence is normally not transcribed. StuPPO9 is the only PPO-like gene model that is not clustered with the others on chromosome 8 , but is independently located on chromosome 2. A number of ESTs were found for StuPPO9, but all of the ESTs were revealed in the cDNA libraries from the tissues of in vitro cultured potato callus (DBLINK ID: LIBEST_015047), abiotic stress treated leaf and root (LIBEST_015048) [25], or pathogen-infected leaf and tuber (LIBEST_008810, LIBEST_009854, LIBEST_ 015324, LIBEST_015920, LIBEST_017649, and LIBEST_
025550) (Additional file 1: Part A and Part C) [26-28]. The expression data of the supporting ESTs of StuPPO9 were mostly not available because the gene model was not annotated previously and most reports focused on annotated genes. However, at least three of the StuPPO9 related ESTs (GenBank ID: CK640809, CO267905 and GT888802) were found to express differentially in the pathogen-infected potato tissues (Additional file 1: Part C). For example, the expression level of the CK640809 was induced 3- to 14-fold higher in potato (cultivar Indira and Bettina) leaf tissue that was inoculated with fungus Phytophthora infestans (See the Table four in reference [27]. The CO267905 showed over 2 -fold induction in the potato (cv. R-gene-free potato clone 386209.10) leaf tissue infected with $P$. infestans at 24 hours post-inoculation (hpi) and its expression level was over 17fold higher at $48 \mathrm{hpi}$ (See the Table one in reference [28]). The relative expression level of GT888802 was about 3-fold higher in potato (cv. Spunta) tubers inoculated with fungus Fusarium eumartii at 24 hpi (Table S2 in reference [26]). These data imply that StuPPO9 is probably an inducible PPO gene expressed in response to disease defense and cell rescue [28].

\section{Generation and selection of amiRNA-expressed transgenic potatoes}

Seven different amiRNAs were designed to target the characterized PPO genes, namely StuPPO1 (previously named POTP1/P2), StuPPO2 (previously, POT32), StuPPO3 (previously, POT33) and StuPPO4 (previously, POT72) in $S$. tuberosum $[1,12]$, with amiRNA sequences complementary to either a specific gene or multiple targets by choosing the appropriate 21-bp region of the corresponding PPO genes (Table 2). The amiRNA sequences were incorporated in an Arabidopsis thaliana miR168a gene backbone built in a plant binary vector (Figure 1), $[29,30]$. From transformation of thousands of explants, 8 to 10 transgenic potato lines for each amiRNA 
Table 2 List of the amiRNA constructs, amiRNA names, amiRNA target sequences and target genes

\begin{tabular}{|c|c|c|c|c|}
\hline Construct & amiRNA name & amiRNA sequence $\left(5^{\prime} \rightarrow 3^{\prime}\right)$ & Target sequence* $\left(5^{\prime} \rightarrow 3^{\prime}\right)$ & Gene name (GenBank accession ID) \\
\hline pPZamiRPPO1 & amiRPPO1 & UUGGUGACUGGUGCAAUUGAC & GUCAAUUGCACCAGUCACCAA & $\begin{array}{l}\text { StuPPO1/POTP1/P2 (XM_006355177/ } \\
\text { M95196/M95197) }\end{array}$ \\
\hline pPZamiRPPO2 & amiRPPPO2 & UUGCUAGCUGGCGGAAGUGAA & UUCACUUCCGCCAGCUAGCAA & StuPPO2/POT32 (U22921) \\
\hline \multirow[t]{2}{*}{ pPZamiRPPO3 } & amiRPPO3 & UUGUUCACUGGGGGGAGUGUA & UACACUCCCCCCAGUGAACAA & POT33 (U22922) \\
\hline & & & UUCACUCCCCCCAGUGAACGA & StuPPO3 (XM_006365320) \\
\hline \multirow[t]{2}{*}{ pPZamiRPPO23 } & amiRPPO23 & UCAUCAACUGGAGUUGAGUUG & CAACUCAACUCCAGUUGAUGA & StUPPO2/POT32 \\
\hline & & & CAACUCAACUCCAGUUGAUGA & StuPPO3/POT33 \\
\hline \multirow[t]{5}{*}{ pPZamiRPPO234 } & amiRPPO234 & UAGAACUCGGAGUUCAACCAA & UUGGUUGAACUCCGAGUUCUA & \\
\hline & & & UUGGUUGAACUCCGAGUUCUU & StuPPO2/POT32 \\
\hline & & & UUGGUUGAACUCCGAGUUCUU & StuPPO3 (XM_006365320) \\
\hline & & & UUGGUUGAACUCUGAGUUCUU & POT33 (U22922) \\
\hline & & & UUGGUUGAACUCCGAGUUCUU & StuPPO4/POT72 (U22923) \\
\hline \multirow[t]{3}{*}{ pPZamiRPPO234A } & amiRPPO234A & AAGAACUCGGAGUUCAACCAA & UUGGUUGAACUCCGAGUUCUU & StuPPO2/POT32 \\
\hline & & & UUGGUUGAACUCUGAGUUCUU & StUPPO3/POT33 \\
\hline & & & UUGGUUGAACUCCGAGUUCUU & StuPPO4/POT72 \\
\hline \multirow[t]{6}{*}{ pPZamiRPPO1234 } & amiRPPO1234 & UCAAGCUCAUUCGCAUUCACA & UGUGAAUGCGAAUGAGCUUGA & \\
\hline & & & UGUGAAUGCGGAUGAGCUUGA & StuPP01/POTP1/P2 \\
\hline & & & UGUGAAUGCAAAUGAGCUUGA & StuPPO2 (XM_006365321) \\
\hline & & & UGUGAAUGCGAAUGAGCUUGA & POT32 (U22921) \\
\hline & & & UGUGAAUGCGAAUGAGCUUGA & StUPPO3/POT33 \\
\hline & & & UGUGAAUGCGAAUGAGCUUGA & StUPPO4/POT72 \\
\hline
\end{tabular}

Note: * The non-identical nucleotides, compared to the target sequence, are marked in bold. The homologous sequences of the predicated PPO-like gene models (StuPPO1 to StuPPO9) to all amiRNA target sequences are aligned in the Additional file 1: Part D.

\begin{tabular}{|c|c|c|c|}
\hline \multirow{2}{*}{$\begin{array}{l}\mathbf{A} \\
M I R \\
168 a(E U 549054.1)\end{array}$} & miR168a & miR168a* & \\
\hline & 5 '- TCGCTTGGTGCAGGTCGGGAA & ATCCCGCCTTGCATCAACTGA & -3 \\
\hline \multirow{2}{*}{$\begin{array}{l}\text { B } \\
\text { pPZamiRPPO1 }\end{array}$} & amiRPPO1 & amiRPPO1* & \\
\hline & TTGGTGACTGGTGCAATTGAC & GTCAATTGCGCCAGTCTCCAT & $35 \mathrm{~S}-\mathrm{T}$ \\
\hline \multirow[b]{2}{*}{ pPZamiRPPO2 } & amiRPPO2 & amiRPPO2* & \\
\hline & TTGCTAGCTGGCGGAAGTGAA & TTCACTTCCACCAGCTTGCAT & $35 \mathrm{~S}-\mathrm{T}-1$ \\
\hline \multirow[b]{2}{*}{ pPZamiRPPO3 } & amiRPPO3 & amiRPPO3* & \\
\hline & TTGTTCACTGGGGGGAGTGTA & TACACTCCCTCCAGTGTACAT & $35 \mathrm{~S}-\mathrm{T}$ \\
\hline \multirow[b]{2}{*}{ pPZamiRPPO23 } & amiRPPO23 & amiRPPO23* & \\
\hline & TCATCAACTGGAGTTGAGTTG & \begin{tabular}{|l} 
CAACTCAACGCCAGTTAATGT \\
\end{tabular} & $35 \mathrm{~S}-\mathrm{T}$ \\
\hline \multirow[b]{2}{*}{ pPZamiRPPO234 } & amiRPPO234 & amiRPPO234* & \\
\hline & $1 / 35 \mathrm{~S}-\mathrm{P} \quad$ TAGAACTCGGAGTTCAACCAA & \begin{tabular}{|l} 
TTGGTTGAATTCCGAGCTCTT \\
\end{tabular} & $35 \mathrm{~S}-\mathrm{T}-1 /$ \\
\hline \multirow{3}{*}{ pPZamiRPPO234A } & amiRPPO234A & amiRPPO234A** & \\
\hline & AAGAACTCGGAGTTCAACCAA & \begin{tabular}{|l|} 
TTGGTTGAATTCCGAGCTCTA \\
\end{tabular} & $35 \mathrm{~S}-\mathrm{T}$ \\
\hline & amiRPPO1234 & amiRPPO1234* & \\
\hline pPZamiRPPO1234 / & $1 / 35 \mathrm{~S}-\mathrm{P}$ TCAAGCTCATTCGCATTCACA & \begin{tabular}{|l} 
TGTGAATGCAAATGAGTTTGT \\
\end{tabular} & $35 \mathrm{~S}-\mathrm{T}$ \\
\hline \multicolumn{4}{|c|}{$\begin{array}{l}\text { Figure } 1 \text { Diagrammatic representation of artificial microRNA constructs. (A) Linear structure of the miR168a primary transcript gene } \\
\text { (MIR168a, nt } 120 \text { to 355, GenBank Accession No. EU549054.1). Sequences of the miR168a and its complementary region (illustrated as } \\
\text { approximately miR168a*) in the gene are displayed in the boxes. (B) Structure of the binary vectors for expression of amiRNAs. Construct names } \\
\text { are indicated at the left. The sequences of the designed amiRNA and its complementary region (approximately amiRNA*) are displayed in the } \\
\text { boxes. 35S-P, CaMV } 35 \text { S promoter. 35S-T, CaMV } 35 \text { S terminator. }\end{array}$} \\
\hline
\end{tabular}


construct were selected and propagated for molecular genetic screening and analysis (Table 3). Significant down-regulation of the targeted gene expression was detected in a number of the resulting transgenic potato lines (Table 3). Based on the initial real-time quantitative reverse transcription PCR (qRT-PCR) assay of the in vitro cultured potato plants, the transcript levels of the StuPPO1 genes were reduced by 68 to $98 \%$ in six amiRPPO1 series transgenic lines (clones). The amiRPPO1 series expressed artificial miRNA - amiRPPO1 designed to target the StuPPO1 gene. Similarly, one amiRPPO2 series line (target: StuPPO2), two amiRPPO3 series lines (target: StuPPO3), five amiRPPO23 series lines (targets: StuPPO2 and StuPPO3), two amiRPPO234 series lines (targets: StuPPO2, StuPPO3 and StuPPO4), three amiRPPO234A series lines (targets: StuPPO2, StuPPO3 and StuPPO4) and five amiRPPO1234 series lines (targets: StuPPO1, StuPPO2, StuPPO3 and StuPPO4) showed substantial reduction of the target gene transcript level(s) (Table 3). Mature amiRNAs were detected by reverse transcription PCR (RTPCR) in the selected amiRPPO1, amiRPPO3, amiRPPO23, amiRPPO234A and amiRPPO1234 series lines (Table 3, Additional file 2: Figure S1). However, no mature amiRNAs were revealed by RT-PCR in the 10 lines of the amiRPPO2 series nor the 10 clones of the amiRPPO234 series (Table 3 and data not shown). The amiRPPO2- and amiRPPO234- transgenic lines were therefore excluded from further evaluations. In addition, small RNA Northern blots were previously done to detect amiRNA expression of the multiple transgenic lines listed in Table 3, including amiRPPO1-7 and -12 (previously named as amiR-POTP1/P2, L7 and L12), amiRPPO2-12, -19 (previously, amiR-POT32, L12 and L19), amiRPPO3-8 and -15 (previously, amiR-POT33, L8 and L15) and amiRPPO2349 and -10 (previously, amiR-POT32/33/72, L9 and L10) [30].

In plants, miRNA-guided RNA silencing has been shown to occur mostly through complementary cleavage of the targeted mRNA by Argonaute proteins [31]. Considering the highly complementary sequences between the designed amiRNAs and their target PPO genes, we used $5^{\prime}$-RACE PCR to detect the possible cleavage of the PPO gene transcript(s). Because of the multiple PPO gene members in potatoes, we developed a strategy for detecting all possible fragmented-mRNA of the PPO genes but not the $5^{\prime}$-capped mRNA (Figure 2A and see the Methods). As predicted, a 253-bp PCR product (including a 45-bp 5'-RACE adaptor) was revealed by the nested PCR round-1 from the enriched poly $(\mathrm{A})^{+}$RNA of the young leaves of line amiRPPO1-12 (Figure 2C). Sequence analysis demonstrated the fragment included two nearly identical sequences differing by one nucleotide (Additional file 3: Figure S2). Both were highly related to the StuPPO1 gene (97 to $99 \%$ identity at nucleotide level) (Additional file 4: Figure S3). The first 10 nucleotides of the 5 '-end of the sequences were complementary to the $5^{\prime}$-end of the designed amiRPPO1, indicating the products were from cleavage of the target StuPPO1 mRNA and the cleavage site was between nucleotides 10 and 11 at the amiRPPO1 site (Figure 2E). The presence of the cleaved StuPPO1 mRNA was also demonstrated by the specific nested PCR-2 (Figure 2D). The results suggested that the expressed amiRNAs in the transgenic plants functioned as the small RNAs that determined the silencing of the gene (s) of interest. The following transgenic lines were selected and propagated for further biological analysis, amiRPPO1$2,-3$ and -12 , amiRPPO3-12 and -15 , amiRPPO23-5, -7 and -9 , amiRPPO234A-4, -6 and -14 , amiRPPO1234$2,-6$ and -12 (Table 3 ).

\section{PPO gene expressions in transgenic potatoes}

No growth abnormities occurred in the amiRNA-expressed transgenic plants under greenhouse conditions. Nor were the range of the tuber sizes and weights significantly different in either the transgenic or the wild types (Data not shown). Relative transcript levels of StuPPO1, StuPPO2, StuPPO3 and StuPPO4 genes in tuber tissues of the transgenic lines were assayed by qRT-PCR with the results illustrated in Figure 3. For lines amiRPPO1-2, -3 and -12 , the expression of the target StuPPO1 gene was suppressed by 90 to $99 \%$. The mRNA levels of the non-targeted StuPPO2 and StuPPO3 in lines amiRPPO1-3 and amiRPPO1-12 were similar to that of the non-transgenic, wild types 'WT', potato controls, but the expression levels of the two genes (StuPPO2 and StuPPO3) in line amiRPPO1-2 were unexpectedly reduced by $50 \%$ and $80 \%$, respectively (Figure 3A). For lines amiRPPO3-12 and -15, the transcript of the target StuPPO3 gene was reduced by over $75 \%$. In addition, the non-targeted StuPPO1 and StuPPO2 gene mRNA levels also decreased by $50-60 \%$ in line amiRPPO3-12, but the two gene transcripts (StuPPO1 and StuPPO2) in line amiRPPO3-15 were close to the level observed in the WT. The non-targeted StuPPO4 gene mRNA was reduced by $\sim 60 \%$ in line amiRPPO3-15 but the same gene mRNA level in line amiRPPO3-12 was almost the same as the WT (Figure 3B). For lines amiRPPO23-5, -7 and -9, the two targeted genes, StuPPO2 and StuPPO3 were almost completely silenced (> 95\%) and the non-targeted StuPPO1 gene was expressed at a level similar to the WT, but the StuPPO4 gene mRNA level was also generally $50-80 \%$ lower (Figure $3 \mathrm{C}$ ). For lines amiRPPO234A-4, -6 and -14 , the mRNA levels of all three targets, StuPPO2, StuPPO3 and StuPPO4 were 75-95\% lower than the WT, but the expression of the non-targeted StuPPO1 gene was also reduced by an average of 50\% (Figure 3D). For lines amiRPPO1234-2, -6 and -12 , the mRNA levels of the targeted StuPPO1, StuPPO2, StuPPO3 and StuPPO4 genes were all suppressed by $85-99 \%$ (Figure 3E). 
Table 3 Screening amiRNA-expressed transgenic potato lines

\begin{tabular}{|c|c|c|c|c|c|c|c|}
\hline \multirow{2}{*}{ Transgenic line } & \multirow{2}{*}{ Kan } & \multirow{2}{*}{ Insert } & \multicolumn{4}{|c|}{ Relative expression level of target gene } & \multirow{2}{*}{ amiRNA } \\
\hline & & & POTP1/P2 & РОT32 & РОT33 & POT72 & \\
\hline amiRPPO1-2 & + & + & $0.02 \pm 0$ & & & & + \\
\hline amiRPPO1-3 & + & + & $0.06 \pm 0$ & & & & + \\
\hline amiRPPO1-7 & + & + & $0.14 \pm 0$ & & & & + \\
\hline amiRPPO1-8 & + & + & $0.03 \pm 0$ & & & & n.t. \\
\hline amiRPPO1-9 & + & + & $0.15 \pm 0.01$ & & & & n.t. \\
\hline amiRPPO1-10 & + & n.t. & n.t. & & & & n.t. \\
\hline amiRPPO1-12 & + & + & $0.34 \pm 0$ & & & & + \\
\hline amiRPPO1-13 & + & n.t. & n.t. & & & & n.t. \\
\hline WT & - & - & $0.99 \pm 0.08$ & & & & - \\
\hline amiRPPO2-1 & + & n.t. & & n.t. & & & - \\
\hline amiRPPO2-4 & + & n.t. & & $1.42 \pm 0.12$ & & & - \\
\hline amiRPPO2-9 & + & + & & n.t. & & & - \\
\hline amiRPPO2-12 & + & + & & $1.03 \pm 0.02$ & & & - \\
\hline amiRPPO2-13 & + & n.t. & & n.t. & & & - \\
\hline amiRPPO2-15 & + & n.t. & & $0.83 \pm 0.11$ & & & - \\
\hline amiRPPO2-16 & + & + & & $0.45 \pm 0.03$ & & & - \\
\hline amiRPPO2-17 & + & n.t. & & n.t. & & & - \\
\hline amiRPPO2-18 & + & + & & $1.07 \pm 0.04$ & & & - \\
\hline amiRPPO2-19 & + & + & & $0.99 \pm 0.03$ & & & - \\
\hline WT & - & - & & $1.00 \pm 0.01$ & & & - \\
\hline amiRPPO3-8 & + & + & & & $1.29 \pm 0.23$ & & + \\
\hline amiRPPO3-9 & + & n.t. & & & n.t. & & n.t. \\
\hline amiRPPO3-12 & + & + & & & $0.66 \pm 0.12$ & & + \\
\hline amiRPPO3-13 & + & n.t. & & & $0.97 \pm 0.17$ & & n.t. \\
\hline amiRPPO3-14 & + & + & & & n.t. & & n.t. \\
\hline amiRPPO3-15 & + & + & & & n.v. & & + \\
\hline amiRPPO3-16 & + & n.t. & & & $1.31 \pm 0.40$ & & n.t. \\
\hline amiRPPO3-17 & + & + & & & n.t. & & n.t. \\
\hline amiRPPO3-18 & + & + & & & n.t. & & n.t. \\
\hline WT & - & - & & & $1.00 \pm 0$ & & - \\
\hline amiRPPO23-3 & + & + & & n.t. & n.t. & & n.t. \\
\hline amiRPPO23-4 & + & n.t. & & $1.03 \pm 0.11$ & $1.28 \pm 0.29$ & & n.t. \\
\hline amiRPPO23-5 & + & + & & $0.32 \pm 0.01$ & $0.44 \pm 0.08$ & & + \\
\hline amiRPPO23-7 & + & + & & $0.28 \pm 0.03$ & $0.19 \pm 0.01$ & & + \\
\hline amiRPPO23-8 & + & + & & $0.42 \pm 0$ & $0.01 \pm 0$ & & + \\
\hline amiRPPO23-9 & + & + & & $0.07 \pm 0.01$ & $0.01 \pm 0$ & & + \\
\hline amiRPPO23-14 & + & n.t. & & n.t. & n.t. & & n.t. \\
\hline amiRPPO23-15 & + & n.t. & & $0.23 \pm 0.07$ & $0.00 \pm 0$ & & n.t. \\
\hline amiRPPO23-16 & + & n.t. & & n.t. & n.t. & & n.t. \\
\hline WT & - & - & & $1.00 \pm 0.08$ & $1.00 \pm 0.12$ & & - \\
\hline amiRPPO234-3 & + & n.t. & & n.t. & n.t. & n.t. & - \\
\hline amiRPPO234-4 & + & n.t. & & n.t. & n.t. & n.t. & - \\
\hline amiRPPO234-5 & + & + & & $0.87 \pm 0.06$ & $0.92 \pm 0.14$ & $1.03 \pm 0.17$ & - \\
\hline
\end{tabular}


Table 3 Screening amiRNA-expressed transgenic potato lines (Continued)

\begin{tabular}{|c|c|c|c|c|c|c|c|}
\hline amiRPPO234-6 & + & n.t. & & $0.98 \pm 0.12$ & $3.39 \pm 0.47$ & $1.50 \pm 0.08$ & - \\
\hline amiRPPO234-7 & + & + & & $1.22 \pm 0.16$ & $1.16 \pm 0.09$ & $1.04 \pm 0.17$ & - \\
\hline amiRPPO234-8 & + & + & & $1.66 \pm 0.02$ & $0.71 \pm 0.04$ & $0.69 \pm 0.02$ & - \\
\hline amiRPPO234-9 & + & + & & $0.47 \pm 0.04$ & $0.40 \pm 0.09$ & $0.50 \pm 0.13$ & - \\
\hline amiRPPO234-10 & + & + & & $0.61 \pm 0.03$ & $0.61 \pm 0.09$ & $0.54 \pm 0.21$ & - \\
\hline amiRPPO234-11 & + & n.t. & & $1.35 \pm 0.16$ & $0.83 \pm 0.17$ & $1.16 \pm 0.13$ & - \\
\hline amiRPPO234-13 & + & n.t. & & n.t. & n.t. & n.t. & - \\
\hline WT & - & - & & $1.00 \pm 0.01$ & $1.00 \pm 0.08$ & $1.00 \pm 0.01$ & - \\
\hline amiRPPO234A-2 & + & n.t. & & $1.09 \pm 0.20$ & $1.53 \pm 0.19$ & $1.26 \pm 0.13$ & n.t. \\
\hline amiRPPO234A-4 & + & + & & $0.34 \pm 0.05$ & $0.43 \pm 0.07$ & $0.04 \pm 0.01$ & + \\
\hline amiRPPO234A-6 & + & + & & $0.09 \pm 0.01$ & $0.02 \pm 0$ & $0.05 \pm 0.01$ & + \\
\hline amiRPPO234A-10 & + & + & & $1.07 \pm 0.03$ & $0.77 \pm 0.25$ & $0.47 \pm 0.02$ & + \\
\hline amiRPPO234A-13 & + & + & & n.t. & n.t. & n.t. & n.t. \\
\hline amiRPPO234A-14 & + & + & & $0.58 \pm 0$ & $0.22 \pm 0.01$ & $0.15 \pm 0.03$ & + \\
\hline amiRPPO234A-15 & + & n.t. & & $1.25 \pm 0.18$ & $0.8 \pm 0.12$ & $1.34 \pm 0.16$ & n.t. \\
\hline amiRPPO234A-16 & + & n.t. & & $0.83 \pm 0.12$ & $1.21 \pm 0.09$ & $0.60 \pm 0.07$ & n.t. \\
\hline WT & - & - & & $0.99 \pm 0.08$ & $1.01 \pm 0.18$ & $0.99 \pm 0.05$ & - \\
\hline amiRPPO1234-1 & + & n.t. & n.t. & n.t. & n.t. & n.t. & n.t. \\
\hline amiRPPO1234-2 & + & + & $0.52 \pm 0.07$ & $0.30 \pm 0.03$ & $0.46 \pm 0.01$ & $0.34 \pm 0.08$ & + \\
\hline amiRPPO1234-3 & + & + & $0.43 \pm 0.04$ & $0.19 \pm 0.06$ & $0.07 \pm 0$ & $0.43 \pm 0.1$ & n.t. \\
\hline amiRPPO1234-6 & + & + & $0.27 \pm 0.03$ & $0.20 \pm 0.04$ & $0.19 \pm 0$ & $0.2 \pm 0.04$ & + \\
\hline amiRPPO1234-7 & + & n.t. & n.t. & n.t. & n.t. & n.t. & n.t. \\
\hline amiRPPO1234-8 & + & n.t. & n.t. & n.t. & n.t. & n.t. & n.t. \\
\hline amiRPPO1234-9 & + & + & n.t. & n.t. & n.t. & n.t. & n.t. \\
\hline amiRPPO1234-11 & + & n.t. & $0.15 \pm 0.01$ & $0.34 \pm 0.11$ & $1.85 \pm 0.17$ & $0.08 \pm 0.01$ & n.t. \\
\hline amiRPPO1234-12 & + & + & $0.28 \pm 0.03$ & $0.66 \pm 0.02$ & $0.47 \pm 0.03$ & $0.18 \pm 0.01$ & + \\
\hline amiRPPO1234-16 & + & n.t. & $0.42 \pm 0.03$ & $0.14 \pm 0.03$ & $0.15 \pm 0$ & $0.08 \pm 0.06$ & n.t. \\
\hline WT & - & - & $1.00 \pm 0.09$ & $1.00 \pm 0.1$ & $1.00 \pm 0.19$ & $1.00 \pm 0.07$ & - \\
\hline
\end{tabular}

Note: Kan, is selection of transgenic lines with Kanamycin (100 mg/L); +, Kanamycin resistant; -, Kanamycin susceptible (such as for WT). Insert, is transgene insertion into the chromosome(s) detected by PCR; +, positive in PCR detection; -, negative in PCR detection; n.t., not test. Relative expression level of target gene, was assayed using in vitro cultured potato leaf and stem tissues by qRT-PCR; the data is the average of three technical repeats and their standard deviation of using one original sample from each transgenic line; n.v., no value; n.t. not test. amiRNA, is detection of mature amiRNAs by RT-PCR; +, detectable (positive); - , not detectable (negative); n.t., not test.

\section{PPO protein level}

Total PPO protein levels in the transgenic and nontransgenic potato tubers were analyzed using a semiquantitative protein dot-blot assay. Figure 4A shows the values of the total PPO protein level in the transgenic potato tuber tissues relative to that in the non-transgenic wild types (relative PPO protein level, simply represented by 'RPR' in this report). The average RPR values for lines amiRPPO1-2, -3 and -12 ranged from 0.70 to 0.77 , indicating total PPO protein levels in these transgenic potato tuber tissues were on average about 23-30\% lower than those in the wild type. Lines amiRPPO3-12 and -15 showed a decline of $15-20 \%$ on average in their total PPO protein level, compared to the wild type. Average reductions of $45-70 \%$ in total PPO protein level were detected in lines amiRPPO23-5, -7 and -9, amiRPPO234A-4, -6 and -14 , based on their average RPRs (0.30-0.55). The average RPRs for lines amiRPPO1234-2, -6 and -12 varied from 0.20 to 0.27 , suggesting that total PPO protein concentrations in these transgenic tubers decreased on average by $73-80 \%$, compared to the wild type (Figure $4 \mathrm{~A}$ ).

\section{PPO enzymatic activity}

Figure $4 \mathrm{~B}$ depicts the PPO enzymatic activities in the transgenic potato tuber tissues relative to those in the wild type (relative PPO activity, abbreviated to 'RPPO' in this report). Based on the RPPOs, the average PPO activity of lines amiRPPO1-2, -3 and -12 was $25-35 \%$ lower than that of the wild type. A reduction of $15-25 \%$ in PPO activity was observed in lines amiRPPO3-12 and -15 . 


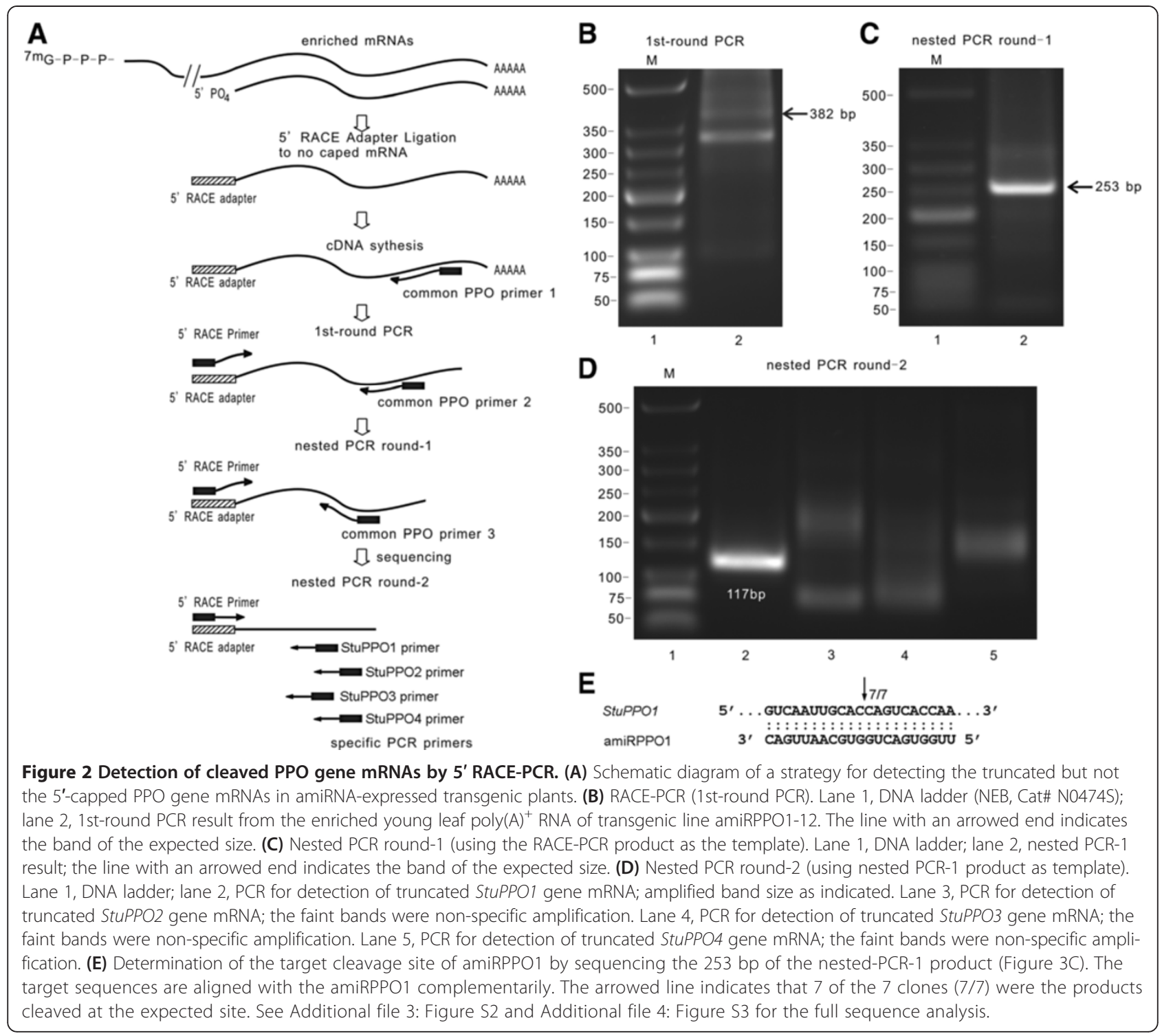

PPO activities in lines amiRPPO23-5, -7 and -9 , amiRPPO234A-4, -6 and -14 , amiRPPO1234-2, -6 and -12 were in a similar range, about $75-95 \%$ less than those of the non-transgenic controls (Figure 4B).

\section{Browning potential and browning phenotype}

Browning was used to measure the potential of phenolic oxidation after mechanical release of PPO proteins from their storage site in plastids [32]. Browning potential of the transgenic potato tuber was compared to that of their non-transgenic wild types (relative browning potential, abbreviated to 'RBR' in this report) and the results are shown in Figure 4C. The RBRs for lines amiRPPO1-2, -3 and -12 , amiRPPO3-12 and -15 ranged between 0.65 and 0.90 , suggesting that browning potentials of these transgenic lines were about 10-35\% lower than those of the WT. Browning potentials of lines
amiRPPO23-5, -7 and -9 , amiRPPO234A-4, -6 and -14 were reduced by $\sim 50-65 \%$ based on their RBR values of $\sim 0.35-0.50$. The ranges of $0.25-0.35$ in RBR for lines amiRPPO1234-2, -6 and -12 indicated that the browning potential of these transgenic lines was about $65-75 \%$ lower than the comparable wild type (Figure 4C). The browning potential results were relatively consistent with the series of visible browning phenotypes displayed after airexposure of the freshly sectioned potato tubers at room temperature (Figure 5). Browning or blackening tissues developed on the sectioned tuber surfaces, typically starting from the vascular ring region and advancing to the medulla with increased exposure time to oxygen in the air. The wild type tubers developed brown tissues more quickly, over a larger area and more severely than in the transgenic tubers. Among the different transgenic types, the amiRPPO1 and amiRPPO3 series lines showed 
Figure 3 Relative transcript levels of PPO genes in transgenic potato tuber tissues. (A) Transgenic lines of series amiRPPO1. (B) Transgenic lines of series amiRPPO3. (C) Transgenic lines of series amiRPPO23. (D) Transgenic lines of series amiRPPO234A. (E) Transgenic lines of series amiRPPO1234. Each column represents the mean value obtained from qRT-PCR performed in triplicate on each biological sample. The bars indicate standard deviation. Two biological replicates (indicated as a and b) from each transgenic line were selected for the assay. Cyclophilin and ef1 a genes were used as normalization references and non-transgenic potatoes (WT) were set as the control.

relatively stronger browning phenotype, followed by the amiRPPO23, amiRPPO234A and amiRPPO1234 series lines, based on the degree of dark color development that ranged from high to low (Figure 5).

\section{Statistical correlations}

Pearson's correlation coefficient $\left(\mathrm{r}^{2}\right)$ analysis indicated significantly strong and positive correlations between RPR, RPPO and RBR. ( $\left.\mathrm{r}^{2}=0.85-0.89, \mathrm{P}<0.0001\right)$ in potato tuber tissues (Table 4). Among the potato PPO genes, the StuPPO2 gene was highly correlated with RPR, RPPO and RBR $\left(r^{2}=0.70-0.80, \mathrm{P}<0.0001\right)$. Both the StuPPO3 and StuPPO4 genes were moderately associated with RPR, RPPO and RBR $\left(r^{2}=0.59-0.71, \mathrm{P}<0.0001\right)$, while the StuPPO1 gene had a weak correlation with the three browning-related parameters, RPR, RPPO and RBR $\left(\mathrm{r}^{2}=0.19-0.27, \mathrm{P}<0.05\right)$ (Table 4).

Principal component analysis (PCA) generated only two principle components with eigenvalues exceeding 1.0 (Kaiser's rule) (Figure 6 and Additional file 5: Table S1). The two components explained $87 \%$ of the total variance. The first principle component (PC1) accounted for $71 \%$ of total variance and had approximately equal positive loading for the variables StuPPO2 gene, StuPPO3 gene, StuPPO4 gene, RPR, RPPO and RBR. Each of the above variables contributed about $14-18 \%$ to the $\mathrm{PC} 1$, suggesting their equivalent proportion in the different transgenic lines. In contrast, the variable StuPPO1 gene also contributed positively to the PC1 but with a lower score ( $<0.8 \%$ contribution). The second principle component (PC2) only accounted for $16 \%$ of the total variance and was mainly influenced by positive loading of StuPPO1 gene (contribution to PC2, 79\%) (Additional file 5: Table S1). The score plot of the $\mathrm{PC} 1$ and $\mathrm{PC} 2$ paralleled the distribution of the browning phenotypes (Figure 6). Lines amiRPPO1-2, -3 and -12 , amiRPPO3-12 and -15 and the WT, susceptible to browning were scattered on the right side of the plot. In contrast, lines amiRPPO23-5, -7 and -9 , amiRPPO234A-4, -6 and -14 , amiRPPO1234-2, -6 and -12 , resistant to browning were separated to the left side of the plot. Noticeably, lines amiRPPO1234-2, -6 and -12, which inhibited StuPPO1, StuPPO2, StuPPO3 and 


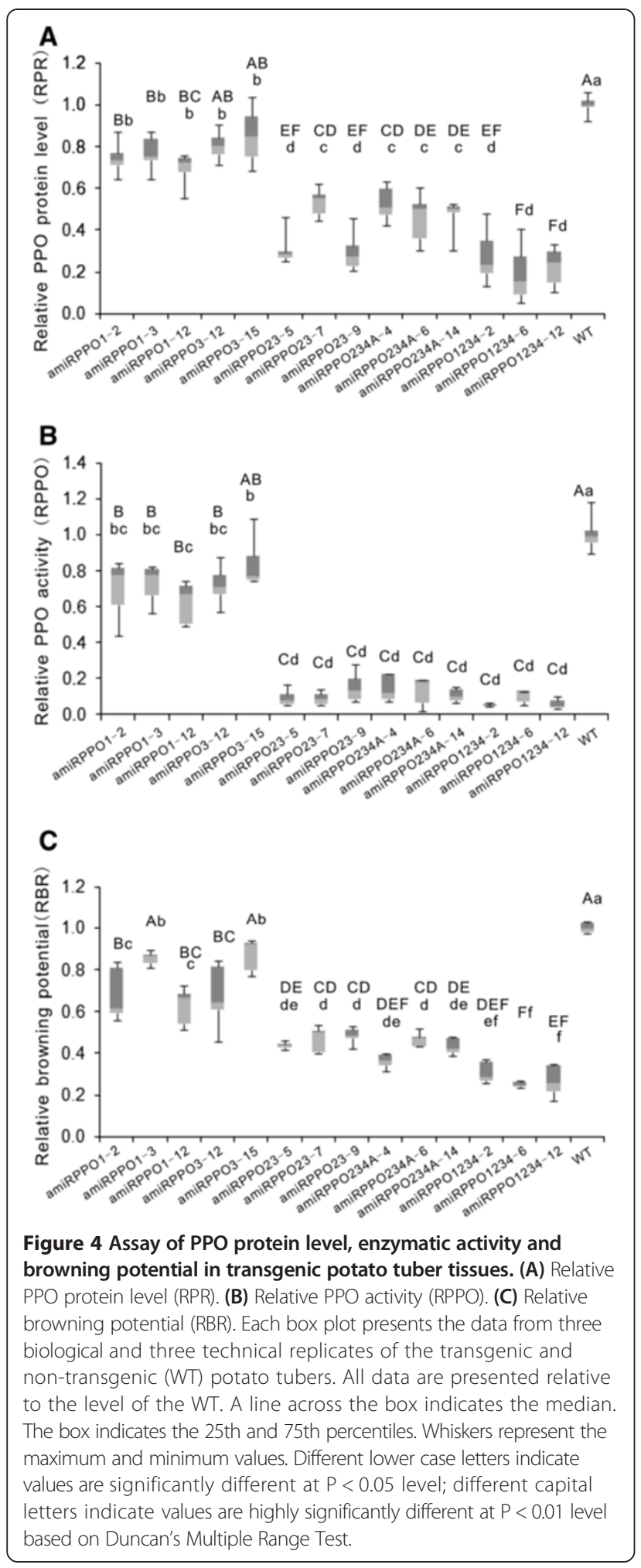

StuPPO4 gene expressions and had the least browning potential, were grouped within the lower quadrant with negative factor scores in both $\mathrm{PC} 1$ and $\mathrm{PC} 2$, and opposite the WT with positive factor scores in both PC1 and PC2.
Hierarchical clustering analysis of the transgenic lines on the variables RPR, RPPO, RBR and PPO gene expression levels produced two major clusters, subcluster-1 (top) and subcluster-2 (bottom) (Figure 7). Members in each of the subclusters displayed a similar pattern with regards to expression trends in the variables RPR, RPPO and RBR. Statistically, subcluster-1 expressed considerably higher values (Min, Max and range) of RPR, RPPO and RBR than subcluster-2 (Figure 4). Interestingly, subcluster-1 consisted the WT and the transgenic lines designed for targeting a single PPO gene (StuPPO1 or StuPPO3), including lines amiRPPO1-2, -3 and -12 , amiRPPO3-12 and -15 , whereas subcluster- 2 included transgenic lines designed for targeting multiple PPO genes, namely, the lines amiRPPO23-5, -7 and -9 , amiRPPO234A-4, -6 and -14, amiRPPO1234$2,-6$ and -12 . The transgenic lines were further divided into smaller sub-groups based on their different scores (Figure 7). For example, the group of lines amiRPPO1-3 and amiRPPO1-12 displayed similar level of StuPPO1 gene suppression but showed almost normal gene transcript levels of StuPPO2, StuPPO3 and StuPPO4 (Figure 3A). Although the gene transcript levels of both StuPPO3 and StuPPO4 in line amiRPPO3-15 were lower by $\sim 70 \%$ than those of the WT (Figure 3B), the two clustered together because the values of RPR, RPPO and RBR in amiRPPO3-15 were closer to those in the WT than other transgenic lines (Figure 4A, B and C). Lines amiRPPO1-2 and amiRPPO3-12 grouped based on their similar gene expression levels of StuPPO2, StuPPO3 and StuPPO4 (Figure $3 \mathrm{~A}$ and $\mathrm{B}$ ) and the generally similar values of RPR, RPPO and RBR (Figure 4A, B and C). The group of lines amiRPPO23-7 and amiRPPO23-9 showed strong down-regulation of both the StuPPO2 and StuPPO3 genes, moderate down-regulation of the StuPPO4 gene (Figure $3 C$ ), as well as similar RPPO and RBR values (Figure $4 \mathrm{~B}$ and $\mathrm{C})$. Three amiRPPO234A series lines $(-4$, -6 and -14 ) clustered together based on their similar down-regulated gene expression levels for StuPPO1, StuPPO2, StuPPO3 and StuPPO4 (Figures 3D), and their similar low values for RPR, RPPO and RBR (Figure 4A, B and $\mathrm{C}$ ). The three amiRPPO234A lines further clustered with line amiRPPO23-5, because the four lines performed similarly in almost all of the variables except that the StuPPO1 mRNA level in the amiRPPO23-5 was moderately higher than in the amiRPPO234A lines (Figures 3C, D, and 4). Three amiRPPO1234 series lines $(-2,-6$ and -12$)$ grouped closely because they had very similar expression trends in all of the 7 variables (Figures $3 \mathrm{E}$ and 4).

\section{Discussion}

PPOs are encoded by a gene family composed of multiple highly conserved gene members in many plant species [2,24]. The differential temporal and spatial 


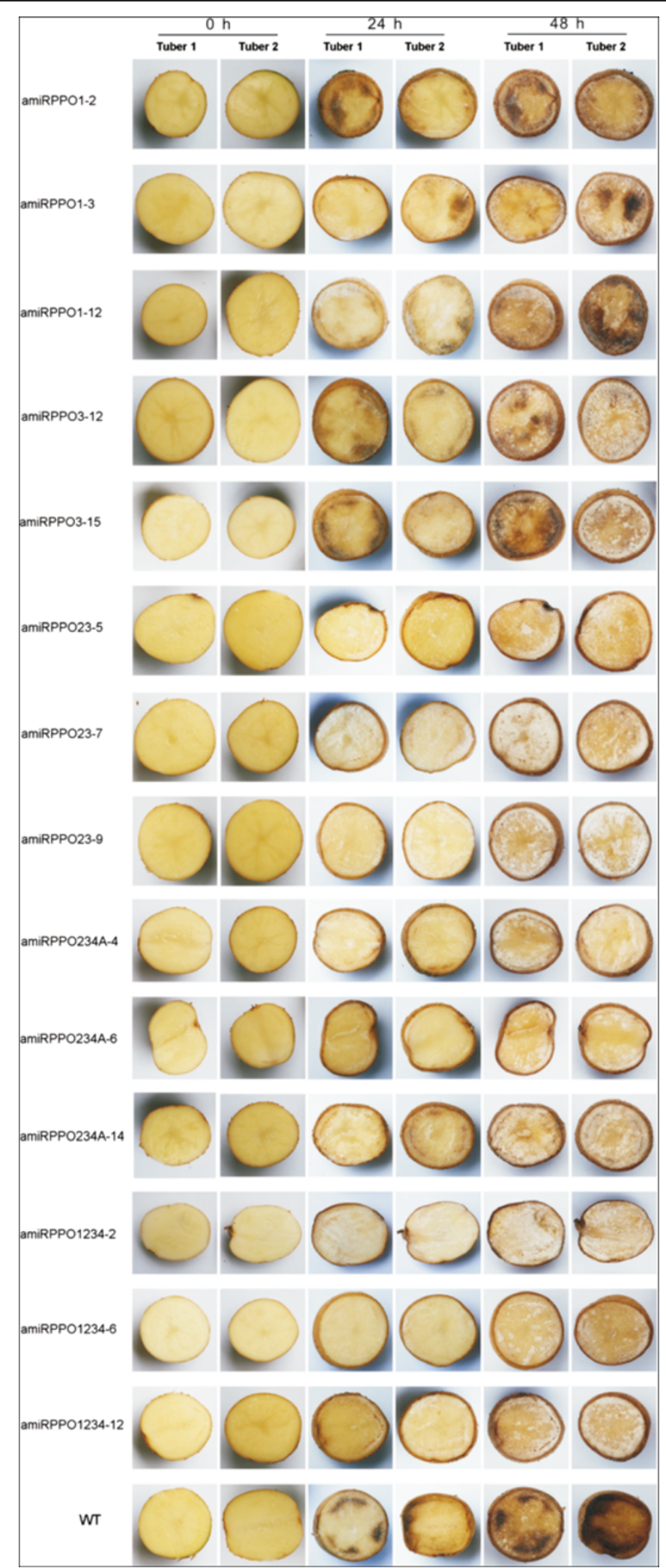

Figure 5 Browning phenotypes. Potato tubers from each transgenic line and wild type were randomly selected, cut into approximately two equal sections and exposed to air/oxygen at room temperature $\left(\sim 25^{\circ} \mathrm{C}\right)$. Images were taken at 0,24 and 48 hour post air exposure. Browning or blackening typically started from vascular ring region and advanced to the medullary tissue with the air-exposure time. 
Table 4 Pearson correlation coefficients analysis results

\begin{tabular}{llll}
\hline & RPR & RPPO & RBR \\
\hline StuPPO1 & 0.20 & 0.19 & $0.27 \mathrm{~d}$ \\
StuPPO2 & $0.70 \mathrm{a}$ & $0.78 \mathrm{a}$ & $0.80 \mathrm{a}$ \\
StuPPO3 & $0.59 \mathrm{a}$ & $0.69 \mathrm{a}$ & $0.71 \mathrm{a}$ \\
StuPPO4 & $0.66 \mathrm{a}$ & $0.69 \mathrm{a}$ & $0.65 \mathrm{a}$ \\
RPR & & $0.85 \mathrm{a}$ & $0.89 \mathrm{a}$ \\
RPPO & & & $0.87 \mathrm{a}$
\end{tabular}

Note: Pearson correlation coefficients $\left(\mathrm{r}^{2}\right)$ of the relationship of PPO gene expression level (StuPPO1, StuPPO2, StuPPO3 and StuPPO4), relative PPO protein expression level (RPR), relative PPO enzymatic activity (RPPO) and relative browning potential (RBR) in potato tuber tissues. $a, P<0.0001$; $d, P<0.05$.

expression patterns of PPO genes in potato, poplar and tomato indicate the functional diversities among the PPO gene members $[1,33,34]$. However, the lack of specific loss-of-function mutants of different PPO genes has impeded progress in understanding the diversified gene functions in the family. Before the present report, several studies reported knockdown of PPO gene expression using siRNA strategies by transformation with hundreds of base pairs of the PPO gene fragments [4-10]. The strategies proved successful in suppression of overall PPO activities and reduction of PPO-mediated browning reactions, but the methods were not able to identify the roles of the individual PPO gene members. The heterogeneous siRNAs

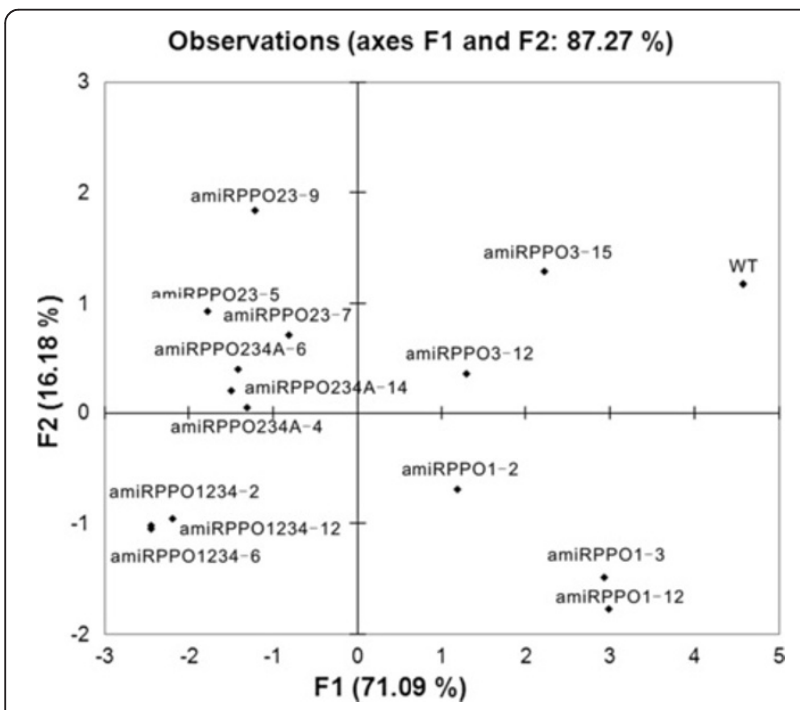

Figure 6 Score scatter plot of the transgenic lines and WT according to principal component analysis (PCA). The bioplot represents the first and second principle components (PC1 and PC2, eigenvalue $>1.0$ ) produced from the PCA of 15 observations (14 transgenic lines and WT) based on seven variables, relative PPO gene expression level of StuPPO1, StuPPO2, StuPPO3 and StuPPO4, RPR, RPPO and RBR. Each line is represented by one dot. Points that are close together correspond to observations that have similar scores for the components displayed in the plot. generated in the transformed plants did not target a specific PPO gene but affected others of the family as well because the PPO gene family shares high homology in nucleotide sequences among its members $[2,24]$. Our data demonstrated that PPO gene isoforms can be suppressed individually or simultaneously using amiRNAs. In addition to significantly down-regulating the PPO genes of interest as predicted in our construct design, several exceptions were observed based on the qRT-PCR assay results (Figure 3). For example, an unexpected moderate to high reduction of the StuPPO2 and StuPPO3 mRNA levels in line amiRPPO1-2, a StuPPO1 gene knockdown transformant, and an unforeseen moderate decrease of the StuPPO1 and StuPPO2 gene transcript copies in line amiRPPO3-12, a StuPPO3 gene knockdown mutant were detected although the analogue regions of the affected genes have 6-8 bp mismatches to the targets of the designed amiRNAs (Figure 3A and B, Additional file 1: Part D). An 8-bp complementarity to the $5^{\prime}$ seed region (canonical 8mer site) [35] of the amiRPPO3 in a StuPPO4 gene sequence region might be related to an unanticipated moderate down-regulation of the non-targeted StuPPO4 gene in Line amiRPPO3-15 (Figure 3B, Additional file 1: Part D). Apart from strong down-regulations of both StuPPO2 and StuPPO3 genes in lines amiRPPO23-5, -7 and -9, StuPPO4 gene mRNA in these lines was at a moderate to low level, suggesting StuPPO4 gene expression was also targeted by amiRPPO23 (Figure $3 \mathrm{C}$ ). The amiRPPO23 was initially designed to target both StuPPO2 and StuPPO3 genes based on incomplete StuPPO4 gene sequence data (GenBank ID: U22923). Later analysis of a StuPPO4 gene related EST sequence (GenBank ID: BG592710) found a region with only one mismatch to the target of amiRPPO23 (position 11, Additional file 1: Part D). A previous study reported mismatches at position 11 were miRNA admissible targets but reduced miRNA-guided cleaving efficiency in vitro and phenotypic effects in vivo [36]. Although the possible target region of the StuPPO1 gene has mismatches at two positions compared to the perfect target sequence of amiRPPO234A (positions 6 and 9 at the amiRPPO234A site, Additional file 1: Part D), the StuPPO1 gene was moderately down-regulated in amiRPPO234A-4, -6 and -14 lines, along with strong down-regulation of the StuPPO2, StuPPO3 and StuPPO4 genes (Figure 3D). It is worth noting that $G: U$ wobble pairing was introduced for targeting StuPPO3 gene in amiRPPO234A and StuPPO1 gene in amiRPPO1234 (Table 2) [37], and the corresponding target genes, StuPPO3 gene in the amiRPPO234A series lines and StuPPO1 gene in the amiRPPO1234 series lines, were indeed down-regulated in both cases (Figure 3D and E). We failed to detect the designed amiRNAs from any of the transgenic plants transformed with the constructs for 


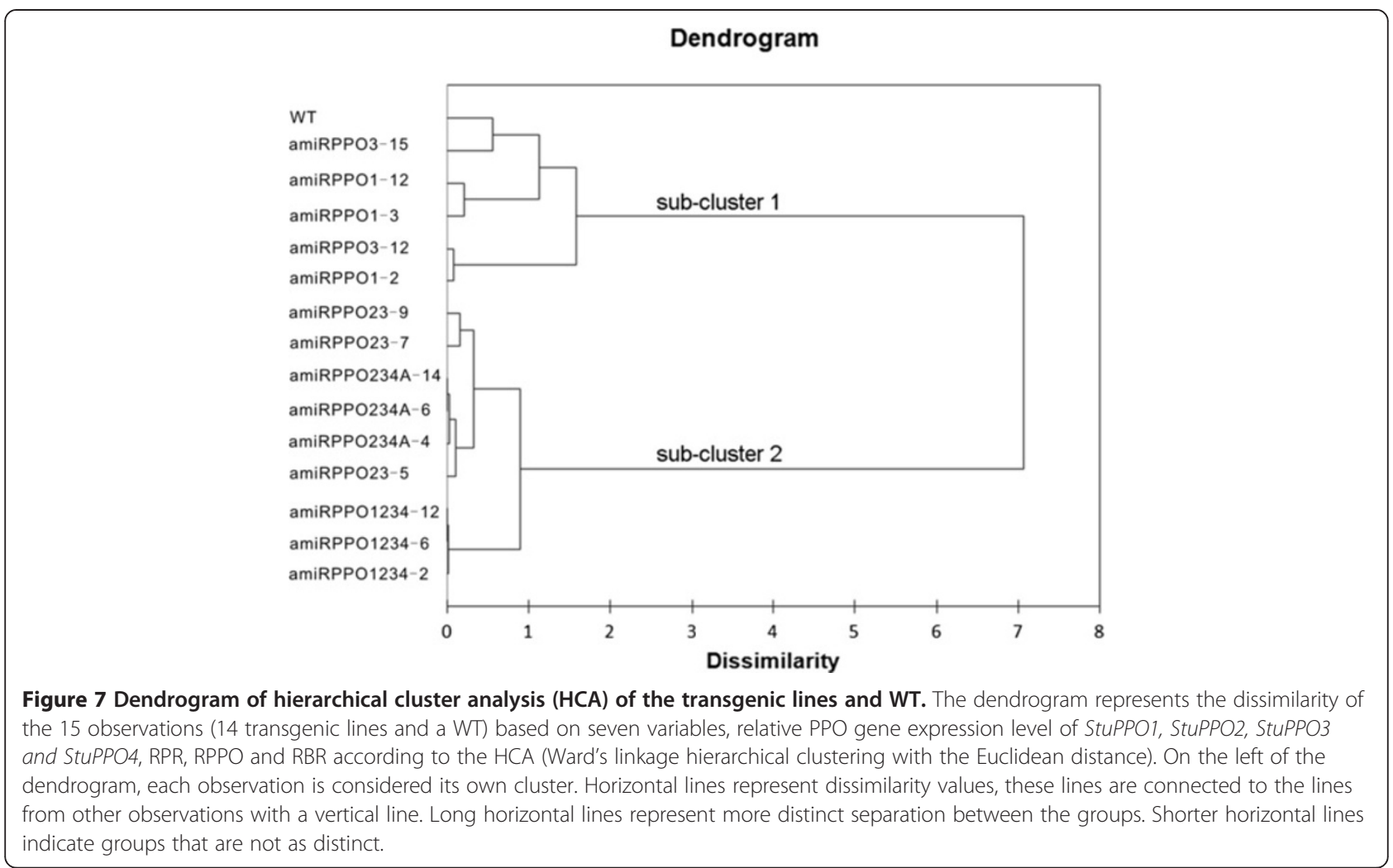

amiRPPO2 (transgenic amiRPPO2 series lines) and amiRPPO234 (transgenic amiRPPO234 series lines) for unknown reasons (Table 3). Investigation of the principles and complexities between amiRNAs and target recognition was beyond the scope of this study. Nonetheless, our data showed that amiRNAs are a potent regulator for modulating expression of potato PPO gene isoforms individually or in combination, and amiRNA targets are more predictable and detectable than the siRNA's. However, not all designed amiRNAs were expressed with similar efficiency in the transgenic plants and off-target effects also occurred occasionally. Appropriate detection methods are required for screening the desired transgenic traits in plants expressing amiRNAs.

Individual or cumulative down-regulation of PPO genes did not usually cause up-regulation of the other PPO genes, except for transgenic line amiRPPO1-12 that showed a reduction of the targeted StuPPO1 gene mRNA level by about $90 \%$ but also an increase of approximately $40 \%$ of the non-targeted StuPPO2 gene transcript (Figure 3A). This implied that different PPO genes may be regulated independently reflecting their diversified functions in the potato plant. Suppression of PPO gene expressions in our transgenic plants resulted in a reduced level of total PPO protein, but the reductions varied depending on the knockdown targets. For example, expression of StuPPO1 was suppressed by 90 to $98 \%$ in lines amiRPPO1-3 and amiRPPO1-12 (Figure 3A), and the total PPO protein level in these two transgenic lines was reduced by $\sim 20$ to $30 \%$ (Figure 4A). Coincidentally, the transgenic lines amiRPPO23-5, -7 and -9, which simultaneously downregulated the StuPPO2, StuPPO3 and StuPPO4 gene (Figure 3C), had reduced total PPO protein level of about $70 \%$ (Figure 4A). Total PPO protein level was reduced by $15 \%$ on average in line amiRPPO3-15 that showed suppression of the StuPPO3 gene (target) and StuPPO4 gene (off-target) (Figures 3B and 4A). These data indicated that $S t u P P O 2$ protein probably contributed $55 \%$ or more to the total PPO protein levels in the non-transgenic potato tuber tissues. The failure to generate StuPPO2-knockdown mutants made it difficult to evaluate the accuracy of the estimation. However, Pearson correlation coefficient indicated that $70 \%$ of the variation in total $\mathrm{PPO}$ protein level in potato tuber tissue was explained by StuPPO2 gene expression $\left(r^{2}=0.70\right.$, Table 3$)$. Analysis of PPO gene expression in the non-transgenic potato tubers by qRT-PCR found that the StuPPO2 gene contributed $67 \%$ to the total PPO gene transcript level, followed by StuPPO1, 28\%, StuPPO3, 4\% and StuPPO4, less than 0.2\% (Additional file 6: Table S2). This also indicated that StuPPO2 is a major contributor to the PPO protein content of the WT potato tubers. Noticeably, StuPPO1, StuPPO2, StuPPO3 and StuPPO4 genes were 
all down-regulated by $90 \%$ or more in transgenic amiRPPO1234-2, -6 and -12 lines (Figure 3E), but the total PPO protein level remained between 20 and 30\% in their tuber tissues. An explanation for this is that the PPO proteins that accumulate in the plastid probably accumulated for a longer duration than the cytosol-produced PPO mRNAs [1,2].

A striking reduction of PPO-mediated browning has been described previously through suppression of overall PPO gene expression using siRNA strategies [4-6]. Here, we have shown that it requires that StuPPO1, StuPPO2, StuPPO3 and StuPPO4 genes were all suppressed simultaneously to achieve optimal inhibition of PPO activity and non-browning phenotype in transgenic potato tuber tissue (Figures 4 and 5), a point not well known or understood previously. It was of particular interest that a series of low browning phenotypes were generated though suppression of the highly conserved PPO gene isoforms when suppressed both individually and in combination using this amiRNA strategy (Figure 5). Analysis of these transgenic clones clearly demonstrated the positive correlations between PPO protein levels (RPR), PPO enzymatic activity (RPPO) and browning potential (RBR) (Figure 4, Table 4). For example, a $\sim 20-30 \%$ reduction at PPO protein level in the tubers of lines amiRPPO1-3 and -12 , where the StuPPO1 gene expression were down-regulated by over $90 \%$, showed a parallel decrease of both PPO activity and browning potential in the tuber tissues. Simultaneous down-regulation of StuPPO1, StuPPO2, StuPPO3 and StuPPO4 genes in lines amiRPPO1234-2, -6 and -12 resulted in $70-80 \%$ loss of tuber PPO protein. As a result of this, PPO enzymatic activity was reduced by $~ 90 \%$ and browning potential by $70-75 \%$. Browning potential correlated better with the PPO protein level than with PPO enzymatic activity. PPO enzymatic activity appeared to decrease relatively faster in our assays than browning reaction (Figure 4). The difference may have resulted from different sample preparation methods used for the two assays. PPO enzymatic activity was more temporally sensitive after the enzymes were mechanically isolated from the plastids, while browning potential recorded the accumulation of pigments in the samples during the time period of the assay. In spite of the small discrepancy between these two data sets, the trend was consistent among the PPO gene down-regulated transgenic plants; the lower the PPO protein level in the potato tuber tissues, the less the PPO activity and browning potential.

Our survey of the recent $S$. tuberosum whole genome database (http://www.jgi.doe.gov) revealed 9 PPO-like gene models (Table 1, Additional file 1). StuPPO1 to StuPPO8 are clustered on chromosome 8 and only StuPPO9 is located on chromosome 2. StuPPO1, StuPPO2, StuPPO3 and StuPPO4 are allelic to previously characterized
POTP1/P2, POT32, POT33 and POT72, respectively, in this report, based on their $95-99 \%$ identities at nucleotide level $[1,12]$. The deduced peptides also show $95-98 \%$ identical positions between the corresponding alleles (Additional file 1). Surprisingly, StuPPO1 is the only possible allele to the POTP1 and the POTP2 genes, and no duplication of the StuPPO1 locus in the $S$. tuberosum genome was discovered. StuPPO5 to StuPPO9 are five novel PPO-like gene models predicted in the $S$. tuberosum genome (Table 1, Additional file 1). However, the low to no prevalence in the EST databases for the StuPPO5, StuPPO6, StuPPO7 and StuPPO8 implies these gene models may be under-transcribed in $S$. tuberosum (Additional file 1: Part C). In addition, the important tyrosinase domains (pfam00264) for PPO enzymatic activity are incomplete in the peptides of the deduced translation of the StuPPO5 and StuPPO7 gene models, suggesting that these two may not be functional like other PPO isoforms (Additional file 1: Part B) [23,24]. StuPPO9 is probably a PPO-like gene involved in disease defense and cell rescue because the ESTs that support for this gene model were dominantly discovered in the tissues of in vitro cultured potato callus, abiotic stress treated leaf and root or pathogeninfected leaf and tuber, and several of the ESTs expressed at significantly higher levels in the pathogen-infected potato tissues (Additional file 1: Part C) [25-28]. Taken together, the number of members of the potato PPO gene family are possibly larger than previously reported, but StuPPO1 (POTP1/P2), StuPPO2 (POT32), StuPPO3 (POT33) and StuPPO4 (POT72) are probably the major developmentally regulated PPO genes in $S$. tuberosum and they are subjected to the targets of the amiRNAs expressed in the transgenic lines. However, extensive experimental studies are required to investigate the existence and function of the potential StuPPO5 to StuPPO9 genes.

\section{Conclusions}

PPO-mediated browning damage is one of the main causes of quality loss in fresh fruit and processed food. It is of great importance and interest to produce crop varieties with low PPO activity for the food industry. Our results have shown that StuPPO1 (POTP1/P2), StuPPO2 (POT32), StuPPO3 (POT33) and StuPPO4 (POT72) genes were the major contributors to the total PPO protein content but the effect of the individuals was not equal and that PPO activity in the tuber tissues paralleled the protein content data. Suppression of expression of one or a few PPO genes did not cause overexpression of the others, but the greatest reduction of PPO activity and the most complete non-browning phenotypes required simultaneous suppression of the expression of StuPPO1, StuPPO2, StuPPO3 and StuPPO4 genes. Our demonstration that PPO gene expression in potato can be suppressed by introduction of 21-nt small RNA regulators provides a new strategy for developing low- or non-browning crops. 
It also suggests that amiRNAs can be applied to silence closely related members of multi-gene family for functional genomics study in non-model plants. In addition, a series of PPO gene knockdown mutants predictably generated provide us important resources for future investigations of the role of PPO genes in functions such as plant development, stress and insect and fungal defense response.

\section{Methods}

\section{Survey of PPO-like genes in Solanum tuberosum}

Nucleotide sequence information of the identified potato PPO genes, including POTP1 (GenBank ID: M95196), POTP2 (M95197), POT32 (U22921), POT33 (U22922), and POT72 (U22923) were obtained from the GenBank. The gene sequences were used as queries to search for the PPO-like gene sequences from a recent $S$. tuberosum whole genome database in the US Joint Genome Institute (http://www.jgi.doe.gov) using the blastn with the default parameters. The BLAST hits were manually checked and the PPO-like gene sequences were retrieved and further analyzed using Vector NTI Advance 11 software (Life Technology, USA), NCBI BLAST platform (blastn, blastp, nr/nt, ESTs, etc.), and Simple Modular Architecture Research Tool (SMART, http://smart.emblheidelberge.de/).

\section{Construction of amiRNA vectors}

The amiRNAs for targeting the characterized potato PPO genes, including StuPPO1 (previously named POTP1/P2), StuPPO2 (previously, POT32), StuPPO3 (previously, POT33) and StuPPO4 (previously, POT72), individually or cumulatively were designed in an Arabidopsis miR-168a gene backbone using an in-house amiRNA designer and constructed as previously described [30]. All constructs were sequenced to confirm the intended construction and the designs. Descriptions of the amiRNAs, their target sequences and genes are listed in Table 2.

\section{Plant genetic transformation}

Transformation of amiRNA constructs with potato cultivar ALT1762 was performed as previously described [30]. Transgenic plants with each of the amiRNA designs were assigned a series of names and numbers as listed in Table 3.

\section{Analysis of PPO gene expression by real-time quantitative reverse transcription PCR (qRT-PCR)}

Potato tissues were disrupted in liquid nitrogen using mortar and pestle. Total RNA was extracted using a Spectrum plant total RNA kit (Sigma-Aldrich, USA) according to the manufacturer's instruction. The purified total RNA samples were treated with Ambion TURBO DNase (Life Technologies, USA) to eliminate potential contamination from genomic DNA, and subsequently re-purified using RNeasy mini kit (Qiagen, Germany). RNA concentration and purity were determined using a ND-1000 spectrophotometer (NanoDrop, Wilmington, DE, USA). First strand cDNA was synthesized from $1 \mu \mathrm{g}$ of the re-purified total RNA using $1 \mu \mathrm{l}$ of RT primer mix (provided with the QuantiTect reverse transcription kit, Cat. No. 205310, Qiagen) and $1 \mu \mathrm{l}$ of SuperScript III reverse transcriptase $(200 \mathrm{U} / \mu \mathrm{l})$ in a $20-\mu \mathrm{l}$ reaction according to the manufacturer's instruction (Life technologies).

Two housekeeping genes, translation elongation factor 1-alpha (ef1 $\alpha)$ and cyclophilin were selected as references for qRT-PCR data analysis. The primer pairs for ef1 $\alpha$ and cyclophilin gene amplicons were the same as the designs by Nicot et al. [38] (Additional file 7: Table S3). The expression stability of the two reference genes in potato tuber tissues was validated by geNorm algorithm [39] (Additional file 8: Figure S4). More than 30 primers for amplions of PPO genes were designed using the Primer 3 (version 0.4.0) [40] based on available gene-specific nucleotide sequence information retrieved from the GenBank databases. Four primer pairs, one for each of the PPO genes, namely StuPPO1, StuPPO2, StuPPO3 and StuPPO4 were eventually selected for qRT-PCR analysis in this study. The PCR amplification specificities using these primer pairs were examined by agarose gel electrophoresis, cloning and sequencing, as well as post-PCR melting curve analysis (Additional file 7: Table S3). All primers used in this research were synthesized by the Integrated DNA Technologies, Inc. (Iowa, USA).

All qRT-PCRs were carried out in triplicate in 96well microplates and performed in a CFX96 real-time PCR detection system (Bio-Rad, USA). Each reaction volume was $20 \mu \mathrm{l}$, comprised of $10 \mu \mathrm{l}$ of Eva green master mix (Applied Biological Materials Inc., Canada), $0.5 \mu \mathrm{M}$ of forward and reverse primers (primer pair mix, $5 \mu \mathrm{l}$ ) and $5 \mu \mathrm{l}$ of appropriately diluted DNA or cDNA template. No-template control (NTC) reactions were included in each plate to monitor potential formation of primer-dimers. The thermal cycling was programed as follows: one initial denaturation cycle at $95^{\circ}$ $\mathrm{C}$ for $10 \mathrm{~min} ; 40$ cycles at $95^{\circ} \mathrm{C}$ for $30 \mathrm{~s}$ and $60^{\circ} \mathrm{C}$ for $30 \mathrm{~s}$. Fluorescence signal was measured at the end of each annealing and extension step at $60^{\circ} \mathrm{C}$. At the end of the qRT-PCR run, a melting curve analysis with a temperature gradient of $0.1^{\circ} \mathrm{C} / \mathrm{s}$ from 65 to $95^{\circ} \mathrm{C}$ was performed to ensure that only single products were produced. Relative expression levels of the PPO gene expressions were calculated based on a method described in Pfaffl [41]. Data normalization was performed using the gene expression values of the ef $1 \alpha$ and cyclophilin in the samples. 
Detection of amiRNAs by reverse transcription PCR (RT-PCR) Detections of mature amiRNAs expressed in the transgenic plants by RT-PCR were conducted as previously described [30]. The forward primers used in the PCR were identical to the corresponding amiRNA sequences except that they were synthesized as oligodeoxynucleotides (Additional file 9: Table S4).

\section{Detection of cleaved mRNA of amiRNA target}

The FirstChoice RLM-RACE kit (Life Technologies) was explored to detect the cleaved mRNA product of the amiRNA target. The kit is designed to amplify cDNA only from full-length, capped mRNA by removing free 5 '-phophates from molecules such as fragmented mRNA using Calf-intestinal alkaline phosphatase (CIAP) before processing to remove the cap structure from full-length mRNA using Tobacco acid pyrophosphatase (TAP). In our application, the CIAP and TAP treatment steps were omitted to prevent full-length, capped-mRNA from ligating to the $5^{\prime}$-RACE adapter (Figure $2 \mathrm{~A}$ ).

Poly $(\mathrm{A})^{+}$RNA was purified using the Dynabead mRNA Purification kit (Life Technologies). Enriched poly(A) ${ }^{+}$ RNA was ligated to the 5 '-RACE adapter provided in the FirstChoice kit without treatment with CIAP and TAP for exclusion of all 5 '-capped mRNA. The conditions for 1 ststrand CDNA synthesis, the followed-up PCR and nested PCR were carried out as instructed by the manufacturer. Besides that, the following primer strategy was developed for detecting possible fragmented mRNA from StuPPO1, StuPPO2, StuPPO3 and StuPPO4 genes (Figure 2A). A reverse primer designed from a conserved sequence region of all four PPO genes was used to synthesize the 1ststrand cDNA. Two other reverse primers designed from another two conserved regions of the four PPO genes were used with the common $5^{\prime}$-RACE primer to conduct the 1st-round PCR and the follow-up nested-PCR-1. Using products from the nested-PCR-1 as the template, nested-PCR-2 reactions were performed with the common 5'-RACE primer and four gene-specific reverse primers, respectively for detecting the bio-origination of the cleaved mRNA products. Distinct bands of appropriate size of PCR fragments were separated by electrophoresis, cloned and sequenced. The oligonucleotides used for detection of cleaved mRNA are listed in Additional file 10: Table S5.

\section{Analysis of PPO protein levels in potato tuber tissues}

A protein dot blot assay was used for semi-quantifying PPO protein levels in potato tubers. Crude protein samples were prepared by powdering $0.2 \mathrm{~g}$ tuber tissue in liquid nitrogen followed by adding $1.6 \mathrm{ml}$ of extraction buffer (2\% SDS, 0.1\% $\beta$-mercaptoethanol in $63 \mathrm{mM}$ Tris-HCl buffer, $\mathrm{pH}$ 6.8). Samples were boiled for $10 \mathrm{~min}$, followed by centrifugation at $20,000 \mathrm{~g}$ for $2 \mathrm{~min}$ to remove insoluble material. The crude protein concentrations were determined using the BCA Protein Assay Kit (Thermo Scientific Pierce, USA) and all samples were then adjusted to $100 \mathrm{mg} / \mathrm{ml}$ protein stock. For dot blot assay, 1:400 diluted samples from the protein stocks were blotted onto polyvinylidene difluoride membrane using Easy-Titer ELIFA System 77000 (Pierce) and probed with a PPO specific antibody (Ab). The PPO $\mathrm{Ab}$ was raised in rabbit against a synthetic peptide (KDWLNSEFFFYDE) corresponding to a conserved region in the deduced StuPPO1, StuPPO2, StuPPO3 and StuPPO4 protein sequences by Applied Biological Materials Inc. (Canada). Two-fold serial dilutions of the synthetic peptide (KDWLNSEFFFYDE) from $50 \mathrm{ng}$ to $0.78125 \mathrm{ng}$ were included in each blot for internal control and making protein standards. The PPO Ab probed blot was incubated with HRP-conjugated anti-rabbit IgG and detected with the Enhanced Chemiluminescence Detection System (GE-Amersham, USA). PPO Protein concentrations were determined using Aida Image Analyzer version 2.00. Relative protein expression level, i.e. PPO proteins in the transgenic plants to that in wild type was calculated for presentation in this study.

\section{PPO enzymatic activity assay}

PPO enzymatic activity was measured using 4-methylcatechol (4-MC) as substrate. A $40 \mathrm{mg}$ sample with a dimension of $2 \mathrm{~mm} \times 5 \mathrm{~mm} \times 5 \mathrm{~mm}$ was collected from the potato tuber tissue next to the skin using a fruit peeler and a single-hole paper punch, and frozen in liquid nitrogen immediately. The sample was homogenized with cold PPO extraction buffer (100 mM sodium phosphate buffer pH 6.0, 2\% TX-100, 2\% PVPP) using a Fast Prep FP120A115 Homogenizer (Thermo Electron Corp, USA). A tenfold diluted extract $(20 \mu \mathrm{l})$ was mixed with $200 \mu \mathrm{l}$ of the assay mixture (50 mM sodium phosphate buffer $\mathrm{pH}$ 6.0, $0.1 \%$ SDS and $15 \mathrm{mM} 4-\mathrm{MC}$ ) in 96-well Microplates. A SpectraMax M2 Microplate Reader (Molecular Devices, USA) was used to measure the absorbance increase at $400 \mathrm{~nm}\left(A_{400 \mathrm{~nm}}\right)$ every 5 seconds for $1 \mathrm{~min}$ at $25^{\circ} \mathrm{C}$. One unit of PPO enzymatic activity was defined as the amount of enzyme necessary to change $A_{400 \mathrm{~nm}}$ in $0.001 / \mathrm{min}$ at $25^{\circ} \mathrm{C}$. The total protein concentration of the extract was detected using the BCA Protein Assay Kit (Pierce) and measured in the same SpectraMax M2 Microplate Reader. The enzymatic activity was calculated as U/mg of total protein.

\section{Browning potential assay}

Browning potential of phenolic compounds in potato tuber tissues was measured using a modification of the method described previously by [32]. Each sample tissue was a collection of two randomly selected potato tubers harvested from the same transgenic line or wild type. 
Triple biological replicates (representing a total of 6 tubers per line) were set up for each treatment. The skins were peeled off before the tubers were chopped into small pieces manually. A 3-gram subsample of each pooled two-tuber replicate was weighed out and homogenized with $15 \mathrm{ml}$ cold sodium phosphate buffer $(50 \mathrm{mM}$, $\mathrm{pH}$ 6.0) using an Ultra-Turrax tissue homogenizer (Takmar, Cincinnati, Ohio) at high speed for $1 \mathrm{~min}$. The homogenate was allowed to oxidize at room temperature for $1 \mathrm{~h}$ before it was vortexed and $1 \mathrm{ml}$ aliquots were centrifuged at $12,000 \mathrm{~g}$ for $10 \mathrm{~min}$. $300 \mu \mathrm{l}$ of the supernatant was loaded in a 96-well microplate for measuring the absorbance at $475 \mathrm{~nm}$ using a SpectraMax M2 Microplate Reader (Molecular Devices) and the $\mathrm{A}_{475 \mathrm{~nm}}$ value was used as an indication of the browning potential.

\section{Statistics analysis}

Analysis of variance with the general linear model procedures, means comparison by Duncan's multiple range test and the Pearson's correlation analysis were performed according to Statistical Analysis System, SAS 9.1 for windows (SAS Institute, Cary, NC). Principal component analysis (PCA) was conducted with XLSTAT 2012 for Windows (Addinsoft, NY). Hierarchical cluster analysis (HCA) was carried out with SYSTAT 12 version 12.02 for Windows (Addinsoft, NY) using Ward's linkage hierarchical clustering with the Euclidean distance.

\section{Additional files}

Additional file 1: Genome-wide analysis of the PPO gene family in Solanum tuberosum. Part A, PPO-like genes predicated based on the $S$. tuberosum genome database; Part B, Confidently predicted domains in the deduced StuPPO1 to StuPPO9 protein sequences using SMART; Part C, List of the supporting ESTs and their biological sources. Part D, List of the amiRNA name, amiRNA sequence, amiRNA target sequence and the target homologous sequences of 9 PPO-like gene models.

Additional file 2: Figure S1. Detection of amiRNAs expressed in transgenic potatoes by reverse transcription PCR (RT-PCR).

Additional file 3: Figure S2. Sequence alignment of the clones of the 5' RACE-PCR product from transgenic line amiRPPO1-12.

Additional file 4: Figure S3. Sequence alignment of the cDNA of the truncated PPO mRNA product from transgenic line amiRPPO1-12 with StUPPO1/POTP1/POTP2.

Additional file 5: Table S1. Results of principal component analysis (PCA) of the transgenic lines and WT.

Additional file 6: Table S2. Relative expression level of PPO genes in the tuber tissue of potato cultivar Alt-1762 (wild type)

Additional file 7: Table S3. Primers and amplicon characteristics of reference genes and PPO genes used in quantification of PPO gene expression in potato tuber tissue measured by qRT-PCR.

Additional file 8: Figure S4. Stability test of candidate reference genes in potato tuber tissue.

Additional file 9: Table S4. List of forward primers used for detection of amiRNAs by reverse transcription PCR.
Additional file 10: Table S5. List of reverse primers used in detection of the cleaved mRNA of amiRNA target in transgenic lines amiRPPO1-12 by 5'-RACE PCR.

\section{Abbreviations}

amiRNA: Artificial microRNA; EST: Expressed sequence tag; PPO: Polyphenol oxidase; qRT-PCR: Real-time quantitative reverse transcription PCR; RACE-PCR: Rapid amplification of CDNA ends PCR; RT-PCR: Reverse transcription PCR; RBR: Relative browning potential; RPPO: Relative PPO activity; RPR: Relative PPO protein level; StuPPO: Solanum tuberosum polyphenol oxidase; WT: Wild type.

\section{Competing interests}

The authors declare that they have no competing interests.

\section{Authors' contributions}

$Y X, W D L, G T, M C, Y S$, and RS conceived and designed the research. GT designed miRNA constructs. $\mathrm{MC}, \mathrm{BB}$, and $\mathrm{YX}$ designed and performed the experiments. $M C, Y X, B B, G T, B D O$ and $P A W$ analyzed the data. $Y X, M C, B B$, and WDL wrote the article. GT, BDO, YS, and RS revised the article. All authors read and approved the final manuscript.

\section{Acknowledgement}

We thank Carl Dubeau and Daniel Piva for partial assistance in maintenance of the transgenic plants used in this study. The systematic nomenclatures of the PPO genes (StUPPOs) and the amiRNAs were suggested by an anonymous reviewer. M. Chi was supported by the Ministry of Education of China and Agriculture and Agri-Food Canada (MOE-AAFC) joint PhD student research program.

\section{Author details}

${ }^{1}$ College of Forestry, Northwest A \& F University, Yangling, Shaanxi, China. ${ }^{2}$ Agriculture and Agri-Food Canada, Pacific Agri-Food Research Centre, Summerland, British Columbia VOH 1ZO, Canada. ${ }^{3}$ Department of Biological Sciences, Michigan Technological University, Houghton, MI 49931, USA.

Received: 23 November 2013 Accepted: 4 March 2014 Published: 11 March 2014

\section{References}

1. Thygesen PW, Dry IB, Robinson SP: Polyphenol oxidase in potato. A multigene family that exhibits differential expression patterns. Plant Physiol 1995, 109(2):525-531.

2. Mayer AM: Polyphenol oxidases in plants and fungi: going places? A review. Phytochemistry 2006, 67(21):2318-2331.

3. Holderbaum DF, Kon T, Kudo T, Guerra MP: Enzymatic browning, polyphenol oxidase activity, and polyphenols in four apple cultivars: dynamics during fruit development. Hort Sci 2010, 45(8):1150-1154.

4. Bachem CW, Speckmann G-J, van der Linde PC, Verheggen FT, Hunt MD, Steffens JC, Zabeau M: Antisense expression of polyphenol oxidase genes inhibits enzymatic browning in potato tubers. Nat Biotechnol 1994, 12(11):1101-1105.

5. Coetzer C, Corsini D, Love S, Pavek J, N T: Control of enzymatic browning in potato (Solanum tuberosum L.) by sense and antisense RNA from tomato polyphenol oxidase. J Agric Food Chem 2001, 49(2):652-657.

6. Murata M, Nishimura M, Murai N, Haruta M, Homma S, Itoh Y: A transgenic apple callus showing reduced polyphenol oxidase activity and lower browning potential. Biosci Biotechnol Biochem 2001, 65(2):383-388.

7. Thipyapong P, Hunt MD, Steffens JC: Antisense downregulation of polyphenol oxidase results in enhanced disease susceptibility. Planta 2004, 220(1):105-117.

8. Wahler D, Gronover CS, Richter C, Foucu F, Twyman RM, Moerschbacher BM, Fischer R, Muth J, Prufer D: Polyphenoloxidase silencing affects latex coagulation in Taraxacum species. Plant Physiol 2009, 151(1):334-346.

9. Richter H, Lieberei R, Strnad M, Novák O, Gruz J, Rensing SA, von Schwartzenberg K Polyphenol oxidases in Physcomitrella: functional PPO1 knockout modulates cytokinin-dependent developmentin the moss Physcomitrella patens. J Exp Bot 2012, 63(14):5121-5135.

10. Richter C, Dirks ME, Gronover CS, Prüfer D, Moerschbacher BM: Silencing and heterologous expression of ppo- 2 indicate a specific function of a 
single polyphenol oxidase isoform in resistance of dandelion (Taraxacum officinale) against Pseudomonas syringae pv. tomato. Mol Plant Microbe Interact 2012, 25(2):200-210.

11. Wesley SV, Helliwell CA, Smith NA, Wang M, Rouse DT, Liu Q, Gooding PS, Singh SP, Abbott D, Stoutjesdijk PA: Construct design for efficient, effective and high-throughput gene silencing in plants. Plant J 2001, 27(6):581-590.

12. Hunt MD, Eannetta NT, Yu H, Newman SM, Steffens JC: cDNA cloning and expression of potato polyphenol oxidase. Plant Mol Biol 1993, 21(1):59-68.

13. Alvarez JP, Pekker I, Goldshmidt A, Blum E, Amsellem Z, Eshed Y: Endogenous and synthetic microRNAs stimulate simultaneous, efficient, and localized regulation of multiple targets in diverse species. Plant Cell 2006, 18(5):1134-1151.

14. Schwab R, Ossowski S, Riester M, Warthmann N, Weigel D: Highly specific gene silencing by artificial microRNAs in Arabidopsis. Plant Cell 2006, 18(5):1121-1133.

15. Tang Y, Wang F, Zhao J, Xie K, Hong Y, Liu Y: Virus-based microRNA expression for gene functional analysis in plants. Plant Physiol 2010, 153(2):632-641.

16. Zhao T, Wang W, Bai $X$, Qi Y: Gene silencing by artificial microRNAs in Chlamydomonas. Plant J 2009, 58(1):157-164.

17. Sablok G, Perez-Quintero AL, Hassan M, Tatarinova TV, Lopez C: Artificial microRNAs (amiRNAs) engineering - On how microRNA-based silencing methods have affected current plant silencing research. Biochem Biophys Res Commun 2011, 406(3):315-319.

18. Schwab R, Voinnet O: RNA silencing amplification in plants: size matters. Proc Natl Acad Sci USA 2010, 107(34):14945-14946.

19. Warthmann $\mathrm{N}$, Chen H, Ossowski S, Weigel D, Herve P: Highly specific gene silencing by artificial miRNAs in rice. PLoS One 2008, 3(3):e1829.

20. Haney CH, Long SR: Plant flotillins are required for infection by nitrogen-fixing bacteria. Proc Natl Acad Sci USA 2010, 107(1):478-483.

21. Zhang X, Li H, Zhang J, Zhang C, Gong P, Ziaf K, Xiao F, Ye Z: Expression of artificial microRNAs in tomato confers efficient and stable virus resistance in a cell-autonomous manner. Transgenic Res 2011, 20(3):569-581.

22. Kim J, Somers DE: Rapid assessment of gene function in the circadian clock using artificial microRNA in Arabidopsis mesophyll protoplasts. Plant Physiol 2010, 154(2):611-621.

23. Marusek CM, Trobaugh NM, Flurkey WH, Inlow JK: Comparative analysis of polyphenol oxidase from plant and fungal species. J Inorg Biochem 2006, 100(1):108-123.

24. Tran LT, Taylor JS, Constabel CP: The polyphenol oxidase gene family in land plants: lineage-specific duplication and expansion. BMC Genomics 2012, 13:395.

25. Rensink W, Hart A, Liu J, Ouyang S, Zismann V, Buell CR: Analyzing the potato abiotic stress transcriptome using expressed sequence tags. Genome 2005, 48(4):598-605.

26. D'Ippólito S, Martín ML, Salcedo MF, Atencio HM, Casalongué CA, Godoy AV, Fiol DF: Transcriptome profiling of Fusarium solani f. sp. eumartii -infected potato tubers provides evidence of an inducible defense response. Physiol Mol Plant Pathol 2010, 75(1-2):3-12.

27. Ros B, Thummler F, Wenzel G: Analysis of differentially expressed genes in a susceptible and moderately resistant potato cultivar upon Phytophthora infestans infection. Mol Plant Pathol 2004, 5(3):191-201.

28. Tian ZD, Liu J, Wang BL, Xie CH: Screening and expression analysis of Phytophthora infestans induced genes in potato leaves with horizontal resistance. Plant Cell Rep 2006, 25(10):1094-1103

29. Yan J, Gu Y, Jia X, Kang W, Pan S, Tang X, Chen X, Tang G: Effective small RNA destruction by the expression of a short tandem target mimic in Arabidopsis. Plant Cell 2012, 24(2):415-427.

30. Bhagwat $B$, Chi M, Su L, Tang H, Tang G, Xiang Y: An in vivo transient expression system can be applied for rapid and effective selection of artificial microRNA constructs for plant stable genetic transformation. $J$ Genet Genomics 2013, 40(5):261-270.

31. Jones-Rhoades MW, Bartel DP, Bartel B: MicroRNAs and their regulatory roles in plants. Annu Rev Plant Biol 2006, 57:19-53.

32. Culley DE, Dean BB, Brown CR: Introgression of the low browning trait from the wild Mexican species Solanum hjertingii into cultivated potato (S. tuberosum L.). Euphytica 2002, 125(3):293-303.

33. Thipyapong P, Joel DM, Steffens JC: Differential expression and turnover of the tomato polyphenol oxidase gene family during vegetative and reproductive development. Plant Physiol 1997, 113(3):707-718.
34. Tran LT, Constabel CP: The polyphenol oxidase gene family in poplar: phylogeny, differential expression and identification of a novel, vacuolar isoform. Planta 2011, 234(4):799-813.

35. Witkos T, Koscianska E, Krzyzosiak W: Practical aspects of microRNA target prediction. Curr Mol Med 2011, 11(2):93.

36. Mallory AC, Reinhart BJ, Jones-Rhoades MW, Tang G, Zamore PD, Barton MK Bartel DP: MicroRNA control of PHABULOSA in leaf development: importance of pairing to the microRNA 5' region. EMBO J 2004, 23(16):3356-3364.

37. Xia Z, Clark P, Huynh T, Loher P, Zhao Y, Chen H-W, Rigoutsos I, Zhou R: Molecular dynamics simulations of Ago silencing complexes reveal a large repertoire of admissible 'seed-less' targets. Sci Rep 2012, 2:569.

38. Nicot N, Hausman J-F, Hoffmann L, Evers D: Housekeeping gene selection for real-time RT-PCR normalization in potato during biotic and abiotic stress. J Exp Bot 2005, 56(421):2907-2914.

39. Vandesompele J, De Preter K, Pattyn F, Poppe B, Van Roy N, De Paepe A Speleman F: Accurate normalization of real-time quantitative RT-PCR data by geometric averaging of multiple internal control genes. Genome Biol 2002, 3(7):research0034

40. Rozen S, Skaletsky H: Primer3 on the WWW for general users and for biologist 692 programmers. Bioinformatics Methods Protoc 2000, 695:365-386.

41. Pfaffl MW: A new mathematical model for relative quantification in real-time RT-PCR. Nucleic Acids Res 2001, 29(9):e45-e45.

doi:10.1186/1471-2229-14-62

Cite this article as: Chi et al:: Reduced polyphenol oxidase gene expression and enzymatic browning in potato (Solanum tuberosum L.) with artificial microRNAs. BMC Plant Biology 2014 14:62.

\section{Submit your next manuscript to BioMed Central and take full advantage of:}

- Convenient online submission

- Thorough peer review

- No space constraints or color figure charges

- Immediate publication on acceptance

- Inclusion in PubMed, CAS, Scopus and Google Scholar

- Research which is freely available for redistribution

Submit your manuscript at www.biomedcentral.com/submit
C Biomed Central 